\title{
Heart Rate monitor based on IP networking
}

\author{
Miguel G. Molina ${ }^{1,2, \star}$, Priscila E. Garzón ${ }^{1, \star \star}$, Carolina J. Molina ${ }^{1, \star \star \star}$, and Juan X. Nicola ${ }^{2, \star \star \star \star}$ \\ ${ }^{1}$ Universidad de Guayaquil, Guayaquil, Ecuador \\ ${ }^{2}$ Escuela Superior Politécnica del Litoral, Guayaquil, Ecuador
}

\begin{abstract}
With the uprising of Internet of Things (IoT) networks, new applications have taken advantage of this new concept. Having all devices and all people connected 24/7 have several advantages in a variated amount of disciplines. One of them is medicine and the e-health concept. The possibility of having a real time lecture of the vital signs of people can prevent a live threat situation. This paper describes the realization of a device capable of measuring the heart rate of a person and checking for abnormalities that may negatively affect the patient's well-being. This project will make use of electronic devices known as microcontrollers, specifically from the Arduino family, enabling us to capture data, and, with the help of a network card and a RJ-45 cable, transfer it to a PC and visualize the heart rate in real time over its assigned IP address.
\end{abstract}

\section{Introduction}

Society is evolving rapidly into a symbiosis with technology. People now have the urge to be connected with the rest of the world, and to have all the information in their hands. This necessity permitted the arrival of new network and technology requirements. IoT born to meet these demands with new network standards, and allowed the possibility to create devices that let us have more information of any kind available online.

One of the most important knowledge branches that can take advantage of IoT is health. The concept of ehealth started with the idea to have online medical consultation. But, without the proper data of a medical valuation, this task could be futile. The need of devices capable of collect medical data and make them available to the professional staff is imperative.

In this project we will build an IP-based heart rate meter, with the purpose of detecting possible health anomalies. This device will be constructed using an Arduino microcontroller kit and an Arduino Ethernet Shield module. Both parts will form provide the main functionality of the meter. A sensor will be installed in order to detect the heart beats and showing them in the LCD along the heart rate.

\section{Methodology}

Given that TCP/IP was less complex to implement than the OSI model (4 layers vs 7 layers) and specially that TCP/IP showed a more efficient approach with only a few prerequisites, TCP/IP was chosen the standard Internet protocol.

\footnotetext{
^e-mail: miguel.molinav@ug.edu.ec,mgmolina@espol.edu.ec

$\star \star$ e-mail: pris_gar@yahoo.com

$\star \star \star$ e-mail: carolina.molinav@ug.edu.ec

$\star \star \star \star$ e-mail: jxnicola@espol.edu.ec
}

When it comes to organize networks via TCP/IP protocols, a set of strategies must be considered through time [1].

As microcontrollers are used along the project, we must define what a microcontroller is: a simplification of a computer on a printed circuit board. Generally speaking, a computer is composed from various parts that work jointly with respect to a central unit, which is the processor. These parts form the whole microcontroller, which is divided in four fundamental parts: the processor, being no more than a random-access memory, a read-only memory, and ports which permit user interaction [2].

For understanding purposes, it must be known that the heartbeat is defined as the number of times that arteries expand and contract as a result of the natural movement of the heart, which may vary for different reasons. That's why it's said that the heart is at rest when someone is relaxed, and when the heart rate increases product of some type of body effort, it is considered a heart reaction in order to provide more oxygen and energy to the body. [3]

Heartbeats can be classified by ranges, being 60-100 beats per minute (bpm) the normal for adults, although it may vary according to a number of factors, including age, resulting in the following heart rates:

- First month of life: 70-90 bpm

- One to eleven months of life: $80-130 \mathrm{bpm}$

- Three to four years of life: $80-120 \mathrm{bpm}$

- Five to six years of life: 75-115 bpm

- Seven to nine years of life: $70-110 \mathrm{bpm}$

- Ten or more years of life: $60-100 \mathrm{bpm}$

- Athletes: 40-60 bpm

It may be possible that irregularities exist among lectures, which may indicate some problems in the internal parts of the body and a possible health risk. That's why 
it is necessary to learn how to identify affections with the following diagnostics: [4]

Deep pulse: Pulse is barely perceptible, which may indicate a blood deficiency.

Fast pulse: Pulse acceleration denotes heat or internal fever. It may be toxemia or an overdose of toxic substances, infection, or even anxiety.

Slow pulse: Indicates lack of energy in athletes, internal cold, or even depression. People with chronic fatigue have this type of pulse.

Wide and tense pulse: The organ is congested.

Small pulse: Also known as a hard and tense pulse, indicates the organ is contracted.

Weak pulse: The organ has low energy.

As we already said, normal heart rate is between 60 and $100 \mathrm{bpm}$. When the heart passes the $100 \mathrm{bpm}$ it's a tachycardia, and if it's below $60 \mathrm{bpm}$, it's a bradycardia. [5][?]

\section{Specifications}

For the development of our project, we researched about:

- Electronic circuits

- Heartbeats

- Heart diseases

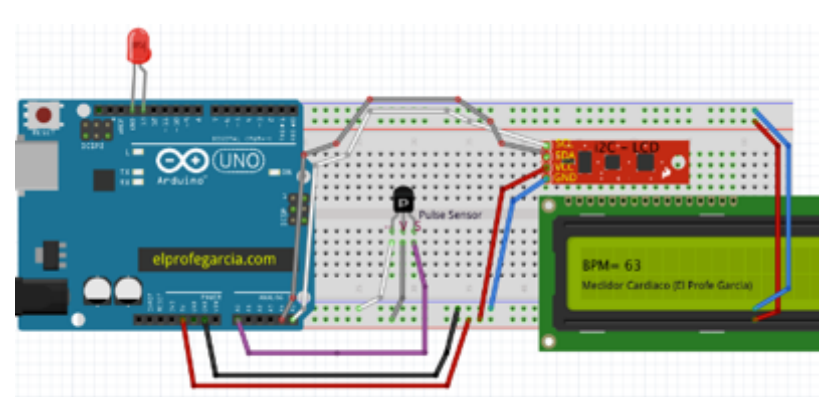

Figure 1. Schematic of the device.

The heart rate monitor has an Arduino UNO as the principal component, with its specs being: [6]

- ATmega328 microcontroller.

- 7-12V input voltage.

- $14 \mathrm{I} / \mathrm{O}$ pins (6 PWM outs).

- 6 analog inputs.

- $32 \mathrm{k}$ Flash memory.

- $16 \mathrm{MHz}$ clock.

We will also uso an Arduino Ethernet Shield, which contains the following indicators and parts:

- RJ-45 standard Ethernet connector and a MicroSD card reader connector.
- Reset button on shield resets both W5100 and Arduino UNO.

- PWR: indicates both the board and shield are fed.

- LINK: indicates the presence of a network link and blinks while sending/receiving data.

- FULLD: indicates a full duplex network connection.

- 100M: indicates the presence of a $100 \mathrm{Mbps}$ network connection (opposed to $10 \mathrm{Mbps}$ )

- RX: blinks when Shield is receiving data.

- TX: blinks when Shield is transmitting data.

- COLL: blinks when collisions are detected in the network.

2x16 LCD

- 16 characters x 2 lined.

- Blue background, white characters

- Backside illumination.

- Parallel interface

I2C controller, which consists of:

- Interface: I2C

- I2C address: 0x27

- Pins: VCC GND SDA SCL

- Voltage feeding: $5 \mathrm{~V}$

- Size: $27.7 \mathrm{~mm}$ by 42.6 millions

- Contrast adjustment: through potentiometer

- Only two I/O interfaces may be used Miscellaneous:

- M-M Cables

- Arduino power cord

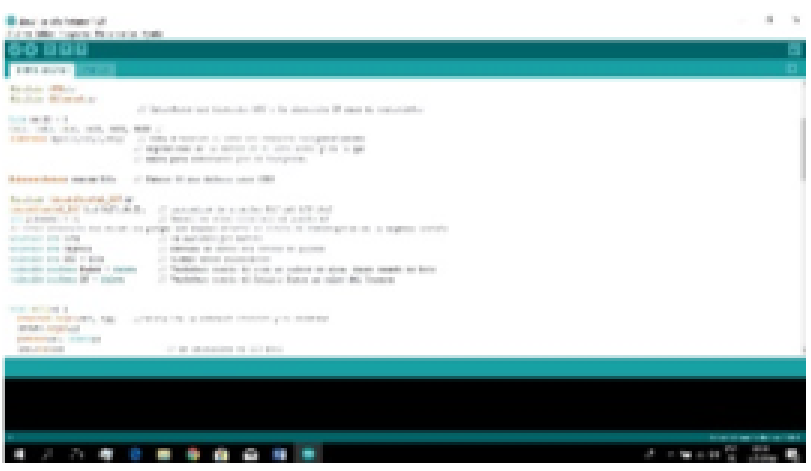

Figure 2. Arduino IDE

\section{Positioning}

After learning about the parts of the heart rate meter, we will proceed with the assembly.

In order to assemble the heart rate monitor, we must get all the necessary parts: Arduino UNO, Ethernet Shield, modem and the heartbeat sensor. 
The process to follow is often the same as in other Arduino-based projects. We will make all connections on a protoboard. We attach both Arduinos between them, and we connect the IC2 and the heartbeat sensor to the Arduino, taking note of the selected pins in order to program the software to be loaded into the Arduino. After connecting the parts to the Arduino, we proceed to connect it to our PC via USB and the Ethernet Shield as a modem in order to have internet connectivity [7]. We open the Arduino suite and check the program code and compile it.

In order to check the results, we open a web browser and enter the selected IP address and it should show all the previously added information, with the configurations made in the Arduino control panel. The sensor will start to work, and we will see the results on the created web page, or in the device's LCD, being the LCD the most up-to-date information due to it being directly connected. If a graphic representation is required, we may use the Arduino-provided serial plotter tool that shows the pulse through time.

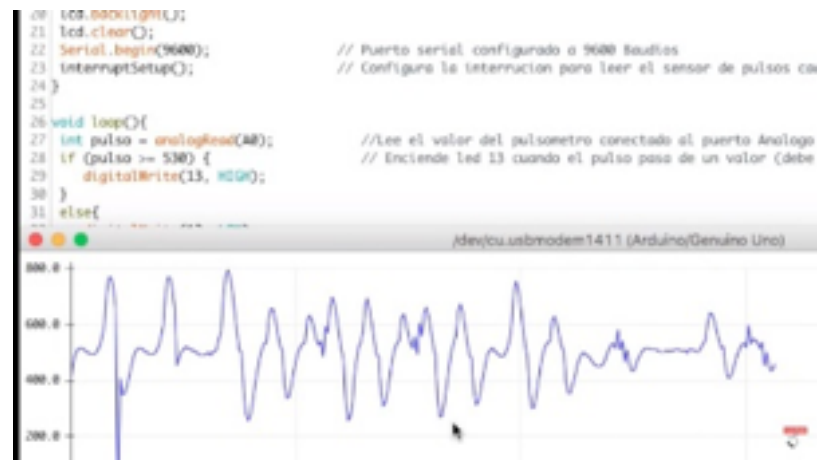

Figure 3. Realtime heart rate graphic

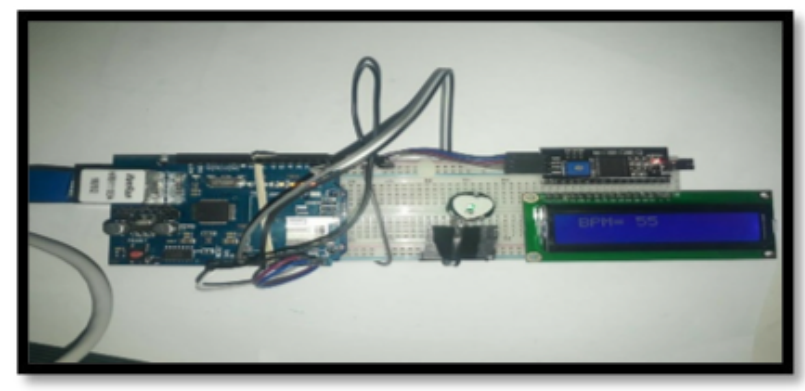

Figure 4. Final assembled circuit

\section{Results}

Once the heart rate monitor is working, we may conclude:

- The maximum heart rate for a healthy person should not exceed 100 bpm, if so, it would be an abnormal condition.

- We obtained a graphic describing the variation of the heart rate though time.

- We obtained a class C IP network from the Arduino and a modem.

- Data can be visualized using a PC as well as a smartphone.

\section{References}

[1] A.L.C. Cabello, Implantación de aplicaciones web en entornos internet, intranet y extranet. IFCD0210 (IC Editorial, 2015)

[2] N. Goilav, L. Geoffrey, Arduino: Aprender a desarrollar para crear objetos inteligentes (Ediciones ENI, 2016)

[3] M. MacGill, ¿Qué es la frecuencia cardiaca? ¿Cuál es la normal? (MedicalNewsToday, 2016)

[4] L. Garcés, Los Pulsos: Diagnóstico de salud en la medicina China (Biomanantial, 2012)

[5] P. Pinheiro, Taquicardia y palpitaciones cardiacas (M.D. Saúde, 2018)

[6] Arduino, Arduino UNO (2018)

[7] MiArduino, Conectar arduino a una red ethernet (2016), http://www. iescamp.es/miarduino/2016/05/11/ conectar-arduino-a-una-red-ethernet/ 